\title{
Budesonide mixed with surfactant did not affect neurodevelopmental outcomes at 6 or 18 months corrected age in observational cohorts
}

\author{
Connie D. Anderson ${ }^{1}$ - T. Brett Kothe ${ }^{1,2}$ - Justin B. Josephsen ${ }^{1}$ - Farouk H. Sadiq ${ }^{1} \cdot$ Nikki Burleyson $^{3}$. \\ Howard L. Williams ${ }^{3} \cdot$ Noah H. Hillman ${ }^{1}$
}

Received: 23 December 2020 / Revised: 10 March 2021 / Accepted: 22 April 2021 / Published online: 13 May 2021

(c) The Author(s), under exclusive licence to Springer Nature America, Inc. 2021

\begin{abstract}
Background The addition of budesonide to surfactant in very-low-birth-weight infants with less severe RDS decreased bronchopulmonary dysplasia (BPD) severity. Long-term neurodevelopmental follow-up was needed to monitor for systemic effects of budesonide.

Methods Infants $\leq 1250 \mathrm{~g}$ who received intratracheal budesonide $(0.25 \mathrm{mg} / \mathrm{kg})$ with surfactant $(n=173)$ were compared to a historical cohort who received surfactant alone $(n=294)$. Peabody Developmental Motor Scales II at 4-6 months corrected age and Bayley Scales of Infant \& Toddler Development III at 18-22 months corrected age were compared.

Results There were no differences in muscle tone or motor skills by Peabody exam. There were no differences in the cognitive, language, or motor domains between cohorts on Bayley III.

Conclusions In a cohort of infants treated with budesonide mixed with surfactant, there were no differences in developmental outcomes at 4-6 months or 18-22 months corrected age.
\end{abstract}

\section{Introduction}

Nearly half of extremely low birth weight infants will require mechanical ventilation and surfactant administration, and many of these infants will develop bronchopulmonary dysplasia (BPD) [1-3]. Lung inflammation is central to the pathophysiology of $\mathrm{BPD}$, and anti-inflammatory medications have been shown decrease BPD rates [4-7]. Delivery of steroids directly to the lungs, with either inhaled budesonide or budesonide instilled with surfactant, have recently been shown to decrease BPD rates and severity [8-12]. Budesonide is a corticosteroid commonly used to treat lung inflammation

Noah H. Hillman

Noah.Hillman@health.slu.edu

1 Division of Neonatal-Perinatal Medicine, Department of Pediatrics, Saint Louis University School of Medicine, Cardinal Glennon Children's Hospital, St. Louis, MO, USA

2 Division of Neonatology, Department of Obstetrics and Gynecology, University of Tennessee Medical Center, Knoxville, TN, USA

3 SSM Health Cardinal Glennon Children's Hospital, St. Louis, MO, USA in asthma and remains primarily in the airways as budesonide esters when used as an aerosolized therapy [13, 14]. However, when mixed with surfactant, the budesonide is transported throughout the distal lung where the alveolar simplification of BPD occurs $[9,15,16]$. The combination of budesonide $(0.25 \mathrm{mg} / \mathrm{kg})$ and surfactant improves gas exchange, matures the lung, and reduces lung inflammation in respiratory distress syndrome (RDS) animal models [9, 15-19]. Unfortunately, budesonide is detected in the plasma of both preterm infants and preterm sheep given budesonide mixed with surfactant [9, 18-20]. Thus systemic effects of budesonide, including in the brain, need to be monitored closely.

Early postnatal steroids have been associated with worse neurologic outcomes, so it is crucial to understand the role of intratracheal budesonide in neurodevelopment [7]. Since BPD and postnatal corticosteroids are both associated with poor neurologic outcomes, exposing preterm infants with a high risk of BPD to short-burst of steroids might decrease inflammation and shift the balance towards improved outcomes $[4,21,22]$. In infants with moderately severe RDS $\left(\mathrm{FiO}_{2}>0.5\right.$ and mechanical ventilation), surfactant with budesonide did not alter neurologic outcome at 18 months corrected age [9]. Infants randomized to prolonged inhaled budesonide had similar long-term neurodevelopmental 
outcomes to controls, but with a slight increase in mortality [8]. We have previously reported a decrease in BPD severity with no increase in mortality with intratracheal budesonide mixed with surfactant [10]. Since our NICU administered budesonide with surfactant to all infants $\leq 1250 \mathrm{~g}$ who failed CPAP, it is critical to evaluate the effects of a potent glucocorticoid on the neurodevelopment of infants with less severe RDS than previous randomized trials $[10,23]$. The infants in our previous observational study were followed clinically in our high-risk follow-up clinic, and we now report available data for motor developmental exams at 6 months corrected age (CA) and neurodevelopmental data at 18-22 months CA.

\section{Methods}

The Division of Neonatology at Saint Louis University and SSM Health Cardinal Glennon Children's Hospital made a local clinical practice change in 2016 to include intratracheal budesonide with pulmonary surfactant due to a high, local BPD rate. Hospital outcomes of the cohorts have been previously published [10]. All patients $\leq 1250 \mathrm{~g}$ are enrolled in our high-risk follow-up clinic, though the percent that are followed is dependent on parental decisions and distance from NICU. Retrospective data collection and analysis of available follow-up data was extracted from the medical record with approval by the Saint Louis University Institutional Review Board.

Beginning on August 1st, 2016, our level III/IV NICUs at SSM Health Cardinal Glennon Children's Hospital and St. Mary's Hospital began to administer budesonide $0.25 \mathrm{mg} / \mathrm{kg}$ (Pulmicort Respules $0.5 \mathrm{mg} / 2 \mathrm{~mL}$, AstraZeneca Pharmaceuticals) mixed with beractant surfactant $4 \mathrm{~mL} / \mathrm{kg}$ (Survanta, $100 \mathrm{mg} / \mathrm{kg}$, AbbVie) to all preterm born infants $\leq 1250 \mathrm{~g}$ who were intubated and would have normally received surfactant alone [10]. Our clinical practice, regardless of gestational age (GA) or weight, is an initial trial of continuous positive airway pressure (CPAP) with bubble CPAP and to utilize noninvasive ventilation failure guidelines similar to the SUPPORT trial $[24,25]$. Very preterm infants who fail CPAP within the first $24 \mathrm{~h}$ of life or who are intubated during the newborn resuscitation receive surfactant. Infants who received the combination of budesonide and surfactant (August 2016 to August 2018) were compared to a historical cohort of infants born $\leq 1250 \mathrm{~g}$ who received surfactant alone (August of 2013 until July of 2016) [10]. Infants born <23 weeks gestation, $<500 \mathrm{~g}$, who died within the first $12 \mathrm{~h}$, or with congenital anomalies were excluded.

\section{Hospital data}

Retrospective data were extracted from the electronic medical record (Epic Systems Corporation) using Microsoft
SQL server and verified via chart review. BPD was defined as the need for oxygen on day of life (DOL) 28 and any level of respiratory support at 36 weeks corrected gestational age (CGA) [26]. BPD was then further subcategorized by severity: 1) No BPD: room air or CPAP with an $\mathrm{FiO}_{2}$ of 0.21 at DOL 28 and no respiratory support at 36 weeks CGA; 2) Mild BPD: respiratory support on DOL 28 and room air at 36 weeks CGA; 3 ) Moderate BPD: $\mathrm{FiO}_{2}$ $>0.21$ but $<0.30$ at 36 weeks CGA; 4) Severe Type 1: $\mathrm{FiO}_{2} \geq 0.30$ or the need for CPAP at 36 weeks CGA; 5) Severe Type 2: non-invasive positive pressure ventilation (NIPPV) or mechanical ventilation at 36 weeks CGA [27]. BPD severity was also categorized based on the grading system outlined by the NICHD Workshop in 2018 [28]. Grade 3 BPD (Severe) included invasive ventilation with $\mathrm{FiO}_{2}>0.21$ or non-invasive respiratory support (CPAP, NIPPV, nasal cannula $>3 \mathrm{~L} / \mathrm{min}$ ) with $\mathrm{FiO}_{2}>0.30$. Intraventricular hemorrhage (IVH) grading and periventricular leukomalacia (PVL) were identified by ultrasound or MRI by board-certified Pediatric Radiologists at 7-10 days after birth and at 36 weeks corrected gestational age. Retinopathy of prematurity (ROP) stage and treatment (laser therapy or bevacizumab) were determined by board-certified Pediatric Ophthalmologists [29].

\section{Long term neurodevelopmental data}

Infants born $\leq 1250 \mathrm{~g}$ typically follow up with our high-risk follow-up clinic at Cardinal Glennon Children's Hospital for oxygen therapy management, nutritional support, and evaluation by Occupation or Physical therapy (OT/PT) and Psychology. We typically can only follow about $80 \%$ of very-low-birth-weight (VLBW) patients due to long-distance referrals to our regional NICU. Patients are assessed by OT/ PT at 4-6 months CA with a modified Peabody Developmental Motor Scales II for fine and gross motor skills [30]. Results are scaled and reported as chronologic age equivalent in months. These values are then compared with the corrected age of the infant at the time of the exam. Some infants also received a second Peabody assessment at 8-10 months CA. At 18-22 months CA, infants are evaluated with the Bayley Scales of Infant Development III by trained Psychologists for the cognitive domain, language domains (receptive and expressive), and motor domains (fine and gross) [3, 23]. The standardized mean Bayley score for each domain is 100 , with a standard deviation of 15 . Bayley scores were labeled abnormal for a domain if the composite score for corrected age was $<85$, and as extremely low for scores of $<70$. A "Combined Cognitive Bayley III score" was calculated as the mean composite scores in the cognitive and language domains for comparison with Bayley II Mental Developmental Indexes (MDI) scores [3]. Infants unable to perform cognitive testing due to severe neurodevelopmental 
Table 1 Cohort demographics and hospital outcomes.

\begin{tabular}{llll}
\hline & Surfactant only & Surfactant and budesonide & $p$ value \\
\hline Cohort Years & August 2013 to July 2016 & August 2016 to August 2018 & \\
Infants in Cohort & 294 & 173 & $0.71^{\mathrm{a}}$ \\
Gestational Age (weeks) & $26.7 \pm 2.1$ & $26.7 \pm 2.1$ & $0.39^{\mathrm{a}}$ \\
Birthweight (g) & $846 \pm 205$ & $863 \pm 214$ & \\
Outcomes during hospitalization associated with Neurodevelopment & $0.52^{\mathrm{b}}$ \\
ROP $\geq$ Stage 2 & $18 \%(52 / 294)$ & $15 \%(26 / 173)$ & $0.90^{\mathrm{b}}$ \\
IVH: Grade III or IV & $16 \%(48 / 294)$ & $16 \%(27 / 173)$ & $0.83^{\mathrm{b}}$ \\
PVL & $5 \%(15 / 294)$ & $6 \%(10 / 173)$ & $0.82^{\mathrm{b}}$ \\
VP Shunt or Reservoir & $4 \%(12 / 294)$ & $5 \%(8 / 173)$ & $0.39 \mathrm{~b}$ \\
Moderate/Severe BPD/Death & $74 \%(218 / 294)$ & $70 \%(121 / 173)$ & $0.03^{\mathrm{b}}$ \\
Severe BPD Type 2 or Death & $19 \%(57 / 294)$ & $12 \%(20 / 173)$ & $0.02^{\mathrm{b}}$ \\
Grade 3 BPD or death [28] & $31 \%(91 / 294)$ & $21 \%(36 / 173)$ & $0.53^{\mathrm{b}}$ \\
Death during initial admission & $10.5 \%(31 / 294)$ & $8.7 \%(15 / 173)$ &
\end{tabular}

Mean \pm SD.

$I V H$ Intraventricular Hemorrhage, $P V L$ Periventricular Leukomalacia, ROP Retinopathy of prematurity, VP Ventriculoperitoneal Shunt, Severe Type 2 BPD Mechanical Ventilation or NIPPV at 36 weeks, Grade 3 $B P D$ Mechanical ventilation or CPAP/NIPPV with $\mathrm{FiO}_{2}>0.30$.

${ }^{\text {a}}$ Unpaired $t$-test.

${ }^{\mathrm{b}}$ Chi-squared. disability were assigned scores of $<70$. Due to Covid-19 precautions, all in person Bayley exams were stopped in March 2020 and thus some infants reached 22 months CA without an exam in the surfactant and budesonide cohort. Neuromuscular exams were extracted from the medical record near 6 months CA (either from follow-up clinic, Pediatrician, Physical Therapy or Neurology notes) and labeled either hypertonic, hypotonic or normal tone. Data on weight and length at 6 months CA plus/minus 2 weeks and 22 months CA plus/minus 1 month were determined through electronic chart review. Visits to the Emergency Department at Cardinal Glennon Children's Hospital or other hospitals in the SSM Health network, and admission to the hospitals during the first 2 years of life were collected. Albuterol use was determined from prescriptions written in the outpatient setting during the first 2 years of life.

\section{Statistical analysis}

Numerical data were analyzed with student's $t$ tests or Mann-Whitney tests when values were not uniformly distributed. Results are displayed as mean \pm standard deviation or median (25th quartile, 75 th quartile). Dichotomous data was analyzed by chi-squared tests with a two-tailed $p$ value $<0.05$ considered significant. When both Peabody and Bayley III scores were available, Pearson correlations were calculated ( $r$ value and $p$ value for correlation) and linear regression performed for best fit line ( $R^{2}$ value reported). Statistics were generated on GraphPad Prism 6, Vassarstats. net, and IBM SPSS.

\section{Results}

The surfactant only cohort (August 2013 to July 2016) had 294 infants and the surfactant and budesonide cohort (August 2016 to July 2018) had 173 infants (Table 1). There were no differences in the birth weights or gestational ages of the infants between cohorts. There were no differences in the rates of severe ROP, intraventricular hemorrhage, periventricular leukomalacia, or need for a VP shunt or reservoir, which are all morbidities that occur in the NICU and are associated with poor neurologic outcomes at 18-22 months CA [31]. Previously reported hospital outcome data are included in Table 1 [10]. Although there were no differences in the number of infants with moderate/ severe BPD or death between the two cohorts, the severity of the BPD shifted to a less severe phenotype. When evaluated with the more traditional definitions of BPD [27], the number of infants with severe type 2 BPD or death were lower in the surfactant and budesonide cohort. Utilizing the newer grading system of BPD, grade 3 BPD or death also decreased in the surfactant and budesonide cohort [28].

\section{Evaluation at 4-6 months corrected age with Peabody motor assessment}

Of the infants in the initial study cohorts, 263 of the 294 infants (89\%) in the surfactant only cohort and 158 of the 173 infants (91\%) in the surfactant and budesonide cohort were alive at 6 months CA (Table 2). A similar percent of infants received Peabody testing between groups at an 
Table 2 4-6 months follow-up data: Peabody exam, muscle tone.
Table 3 Bayley exam results at 18 months corrected age.

\begin{tabular}{llll}
\hline & Surfactant only & $\begin{array}{l}\text { Surfactant and } \\
\text { Budesonide }\end{array}$ & $p$ value \\
\hline Infants Alive at 6 Months & $263(89 \%)$ & $158(91 \%)$ & $0.53^{\mathrm{b}}$ \\
Infants with Peabody Evaluation (,$\%)$ & $184(70 \%)$ & $97(62 \%)$ & $0.09^{\mathrm{b}}$ \\
Corrected Age at Peabody (months) & $5.2 \pm 1.2$ & $5.0 \pm 1.4$ & $0.24^{\mathrm{a}}$ \\
Peabody Fine Motor (months) & $4.6 \pm 1.4$ & $4.5 \pm 1.1$ & $0.22^{\mathrm{a}}$ \\
Individual's Fine Motor delay (months) & $-0.73 \pm 1.34$ & $-0.63 \pm 1.2$ & $0.62^{\mathrm{a}}$ \\
Peabody Gross Motor (months) & $4.7 \pm 1.2$ & $4.4 \pm 1.2$ & $0.30^{\mathrm{a}}$ \\
Individual's Gross Motor delay (months) & $-0.61 \pm 1.2$ & $-0.50 \pm 1.2$ & $0.49^{\mathrm{a}}$ \\
Motors Concerns noted by evaluator or parents at & $40 \%(73 / 184)$ & $42 \%(41 / 97)$ & $0.76^{\mathrm{b}}$ \\
Peabody & & & $0.24^{\mathrm{b}}$ \\
Abnormal Muscle Tone at 6 months on any note in & $19 \%(46 / 236)$ & $14 \%(20 / 141)$ & \\
medical record (\%) & & &
\end{tabular}

Mean \pm SD.

${ }^{a}$ Unpaired $t$-test.

${ }^{\mathrm{b}}$ Chi-squared.

\begin{tabular}{llll}
\hline & Surfactant only & Surfactant and Budesonide & $p$ value \\
\hline Infants Alive at 18 Months & $260(88 \%)$ & $158(91 \%)$ & $0.35^{\mathrm{b}}$ \\
Infants with Bayley Evaluation (\%) & $161(62 \%)$ & $85(54 \%)^{\mathrm{a}}$ & $0.12^{\mathrm{b}}$ \\
Gestational Age of Bayley infants (weeks) & $26.6 \pm 2.0$ & $26.6 \pm 1.9$ & $0.90^{\mathrm{c}}$ \\
Birth weight of Bayley infants (g) & $850 \pm 194$ & $845 \pm 199$ & $0.85^{\mathrm{c}}$ \\
Corrected Age at Bayley (months) & $18.9 \pm 3.1$ & $18.2 \pm 1.4$ & $0.06^{\mathrm{c}}$ \\
Cognitive Domain Score & $91 \pm 15$ & $93 \pm 14$ & $0.27^{\mathrm{c}}$ \\
Language Domain Score & $83 \pm 15$ & $85 \pm 15$ & $0.36^{\mathrm{c}}$ \\
Motor Domain Score & $86 \pm 16$ & $87 \pm 17$ & $0.53^{\mathrm{c}}$ \\
Infants with 1 or more domains $<70$ & $23 \%(39 / 166)$ & $21 \%(18 / 85)$ & $0.75^{\mathrm{b}}$ \\
Infants with 1 or more domains $<85$ & $62 \%(103 / 166)$ & $58 \%(49 / 85)$ & $0.58^{\mathrm{b}}$ \\
Combined Bayley III score $<80$ & $29 \%(48 / 166)$ & $24 \%(20 / 85)$ & $0.54^{\mathrm{b}}$ \\
\hline
\end{tabular}

Mean \pm SD.

${ }^{a}$ COVID 19 restrictions decreased Bayley testing.

${ }^{\mathrm{b}}$ Chi-squared.

${ }^{\mathrm{c}}$ Unpaired $t$-test. average CA of 5 months. Both cohorts had a delay in their fine motor and gross motor skills by slightly more than a half a month, and there were no differences between the groups. About $40 \%$ of parents or PT/OT evaluators were concerned about motor skills at 5 months $\mathrm{CA}$ in both groups. The number of infants with abnormal muscle tone was similar between groups, noted on a physical exam at 6 months CA in $19 \%$ of the surfactant only cohort and $14 \%$ of the surfactant and budesonide cohort $(p=0.24)$.

\section{Bayley III exams on infants at 18-22 months corrected age}

A similar percent of infants in both cohorts were alive at 18 months CA ( $88 \%$ in the surfactant cohort and $91 \%$ in the surfactant and budesonide cohort, $p=0.30$ ) (Table 3).
Bayley exams were completed in a similar percent of both cohorts (62\% in the surfactant only cohort and 54\% in the surfactant and budesonide cohort). The COVID pandemic decreased the number of infants able to be evaluated in person with Bayley III testing in the surfactant and budesonide cohort. Of the infants who received Bayley testing, there were no differences in the gestational ages or birth weights of the infants between cohorts. The infants were evaluated at roughly 18 months CA in both groups. There were no differences in the cognitive, language, or motor domain scores between the cohorts (Table 3). A similar percent of infants had severe impairment with at least one domain score $<70$ ( $24 \%$ vs $21 \%, p=0.63)$, and one or more domain with scores $<85$ (64\% vs $58 \%, p=0.34)$. For the cognitive domain, $24 \%$ of infants in the surfactant only cohort and $21 \%$ of infants in the surfactant and budesonide 

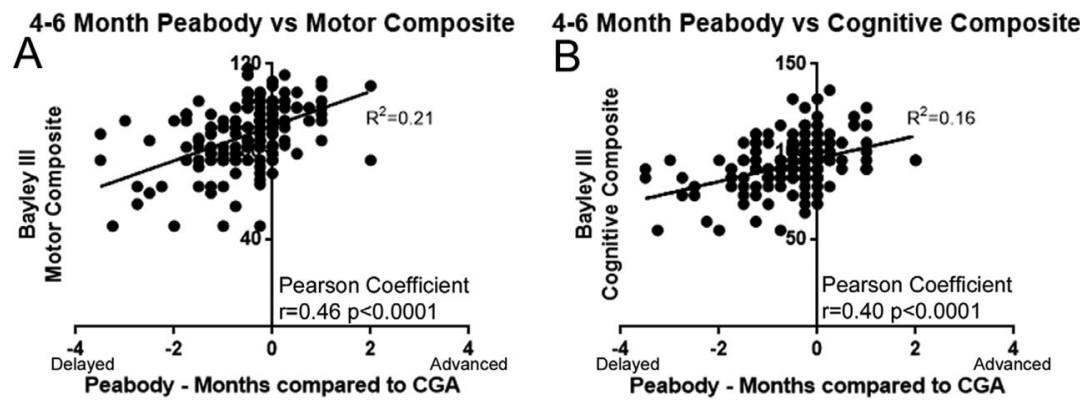

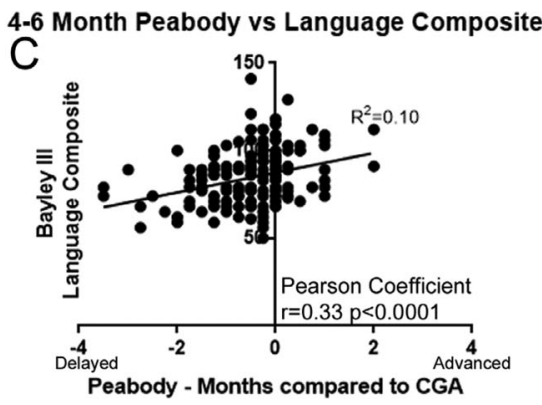

Fig. 1 Correlation of Bayley III scores with Peabody II scores. A Peabody II scores at 5 months, represented as average month delay/ advance (fine and gross motor) for corrected age, show a moderate positive correlation with Bayley III motor scores at 18 months CA $(r=0.46, p<0.001)$. B Peabody II scores correlated slightly less well with cognitive scores $(r=0.40, p<0.001)$. C Peabody II scores had a significant, but minimal correlation with language scores at 18 months CA $(r=0.33, p<0.001)$. Line represents linear regression for best fit with $R^{2}$. Pearson Correlation coefficient $r$ calculated for each component. cohort had scores $<85$ ( $p=0.63)$. For the motor component, $38 \%$ of the surfactant only cohort and $39 \%$ of the surfactant and budesonide cohort had scores $<85(p=0.89)$. And for the language component, $52 \%$ of the surfactant only cohort and $55 \%$ of the surfactant and budesonide cohort had scores $<85$ $(p=0.78)$. The combined Bayley III (average language and cognitive) scores were similar between cohorts, and the percent of infants with scores $<80$ were similar between cohorts. The infants with severe BPD types 1 and 2 had worse neurologic outcomes than patients with no/mild/moderate BPD. The cognitive scores were lower in infants with severe BPD $(89 \pm 15)$ than in mild/moderate BPD $(95 \pm 15)(p<0.001)$, as were language $(82 \pm 14$ vs $86 \pm 16, p=0.04)$ and motor $(84 \pm 17$ vs $90 \pm 13, p<0.001)$ scores. There were no differences between the cohorts when Bayley scores were divided into BPD severity categories (data not shown).

\section{Comparison of neurodevelopmental evaluations at 6 months and 18-22 months}

To evaluate the ability to predict Bayley III scores at earlier ages, each infant's Peabody II exam and neurologic exam at 6 months CA were correlated with their Bayley III scores at 18 months CA. Since no differences were found between cohorts, infants were combined into one analysis. This produced 219 infants from both cohorts that had a documented neurologic exam at 6 months $\mathrm{CA}$ and a Bayley exam at 18-22 months CA. In infants with an exam noting abnormal tone at 6 months CA, 71\% (30/42) of infants later had a Bayley Motor Composite score <85 and 29\% (12/42) had a motor score $>85$. Of infants with a normal motor exam, 33\% (59/177) later had a motor composite score $<85$ and $67 \%(118 / 177)$ had a motor score $>85$. When the average Peabody score at 4-6 months CA, represented as month delay/advance, was compared to the Bayley scores at 18 months CA (Fig. 1), there was only a modest correlation between the two. The Peabody exam had a Pearson correlation coefficient of $0.46(p<0.0001)$ with the motor domain on Bayley III, a coefficient of $0.40(p<0.0001)$ with the cognitive domain, and a $0.33(p<0.0001)$ with the language domain. For the different motor components at 4-6 months CA, there was a similar modest correlation of $0.32(p<0.0001)$ between the Peabody and Bayley for fine motor and $0.43(p<0.0001)$ for gross motor. A few infants $(n=15)$ received Peabody evaluations at 8-10 months CA, and these had a higher positive correlation to the Bayley at 18 months CA: a fine motor correlation of $0.62(p=0.02)$ and a gross motor correlation of $0.57(p=0.03)$.

\section{Other follow-up data}

The weights and lengths at 6 months CA and 20 months CA were similar between the two cohorts (Table 4). The number of infants prescribed albuterol outside the hospital within the first two years of life was slightly smaller in the surfactant and budesonide group (44\% in surfactant only cohort and $33 \%$ in the surfactant and budesonide cohort, $p=0.024)$. The percent of infants with visits to the emergency room and the number of visits were similar between the two groups. The percent of infants hospitalized in the first 2 years were similar between cohorts (47\% vs $38 \%$, $p=0.09$ ), as were the number of hospitalizations.

\section{Discussion}

The addition of budesonide $0.25 \mathrm{mg} / \mathrm{kg}$ to surfactant for the treatment of respiratory distress syndrome was associated with a decrease in the severity of BPD compared with a historical cohort of infants who received only surfactant. There were no differences in the neurodevelopmental assessment of the two cohorts at 5 months CA on Peabody exam or neurologic exams near 6 months CA. Similar scores were obtained on the Bayley III assessment for 
Table 4 Other follow-up data.

\begin{tabular}{llll}
\hline & Surfactant only & Surfactant and Budesonide & $p$ value \\
\hline Weight at 6 months (kg) & $5.3 \pm 0.8$ & $5.5 \pm 0.9$ & $0.15^{\mathrm{a}}$ \\
Length at 6 months (cm) & $56.4 \pm 3.0$ & $56.4 \pm 3.3$ & $0.75^{\mathrm{a}}$ \\
Weight at 20 month (kg) & $10.1 \pm 1.6$ & $10.0 \pm 1.5$ & $0.46^{\mathrm{a}}$ \\
Length at 20 months (cm) & $78.2 \pm 3.8$ & $77.2 \pm 4.3$ & $0.07^{\mathrm{a}}$ \\
Prescribed Albuterol in first 2 years (\%) & $44 \%(115 / 261)$ & $33 \%(52 / 160)$ & $0.024^{\mathrm{b}}$ \\
\% with ER visits in first 2 years & $61 \%(159 / 261)$ & $63 \%(101 / 160)$ & $0.68^{\mathrm{b}}$ \\
Average Number of ER visits & $3.1 \pm 2.2$ & $3.4 \pm 2.6$ & $0.39^{\mathrm{a}}$ \\
\% Hospitalized in first 2 years & $47 \%(122 / 261)$ & $38 \%(61 / 160)$ & $0.09^{\mathrm{b}}$ \\
Average Number of Hospitalizations & $2.0 \pm 1.4$ & $2.1 \pm 1.5$ & $0.50^{\mathrm{a}}$ \\
\hline
\end{tabular}

Mean \pm SD.

${ }^{a}$ Unpaired $t$-test.

${ }^{\mathrm{b}}$ Chi-squared.

cognitive, language and motor domains at 18 months CA. Hospitalizations and emergency room visits were similar between the two cohorts, but there was slightly less use of albuterol in the surfactant and budesonide cohort. Although these infants were part of an observational cohort and follow-up was based on parental participation in our highrisk follow-up clinic, the lack of a difference between treatment cohorts gives additional safety information for NICUs incorporating surfactant and budesonide into their clinical practice. Large, randomized trials (NICHD Budesonide in Babies (BiB) trial (NCT04545866) and the Australian/New Zealand trial Preventing Chronic Lung Disease in Extremely Preterm Infants Using Surfactant + Steroid (PLUSS) (ACTRB12617000322)) are underway to fully evaluate this promising new therapy for decreasing the rates of BPD.

The Peabody exam is often done at 5-6 months CA to assist with qualification for early intervention services for developmental delays. The Peabody is able to discern motor abnormalities, but it is not extremely predictive of outcome at 18-22 months CA on the Bayley exam. It had a stronger correlation for the few babies tested at 8-10 months CA. The Peabody II has been shown to have a poor correlation with the Bayley II at 12 months CA, but this correlation improved with the updated Bayley III exam [30, 32]. When the Peabody was administered at the same time as the Bayley at 18 months CA it had a strong correlation for gross motor $(r=0.88)$ and fine motor $(r=0.77)$ [33]. Early intervention improved neurodevelopment outcomes [34]. There are positive correlations with gross skills and cognition in toddlers, so focusing resources on developmental physical therapies may be useful [35]. Some of the lack of correlation between the Peabody II at 5 months CA and the Bayley exams could be due to the fact that infants who show delays at 6 months CA receive additional early intervention activities, whereas normally developing infants could miss out on these activities. Although the correlations were not very strong, the Peabody exam may be useful for helping stress the need for services with parents or providers.

There were no differences between the two cohorts in any of the three domains studied (cognitive, language, motor) of the Bayley Scales of Infant Development III at 18 months CA. Our findings are similar to the randomized trials with intratracheal budesonide by $\mathrm{Yeh}$ et al. and inhaled budesonide by Bassler et al. which demonstrated no neurodevelopmental differences between budesonide exposed infants and controls [8,9]. The infants in our cohorts were of a similar size and gestational age to the infants in other budesonide studies. Both previous budesonide studies used the Bayley Scales Version II, so direct comparison is more difficult with our observational study. The Bayley III may underestimate the neurodevelopmental outcome compared with the Bayley II exam, as less infants score a value $<70$ on the Bayley III [36]. A Combined Cognitive Bayley III score, which averages the cognitive and language score, of $<80$ correlates moderately well with a Bayley II MDI $<70$ [3]. The percent of infants in our cohorts with a combined Bayley III score $<80$ (29\% in surfactant cohort and $24 \%$ in surfactant and budesonide cohort) were similar to the $21 \%$ of infants in Yeh study with a Bayley II MDI $<70$. In a NICHD network analysis of the Bayley III, $19 \%$ of infants had one domain with a score $<70$ and $34 \%$ had at least one domain score $<85$ [37]. Our data similarly showed $21 \%$ of infants with one domain with a score $<70$ at $21 \%$. Our cohorts' percentage of infants $<85$ $(58 \%)$ for the cognitive and motor domains are slightly higher than NICHD data, but were similar to recently reported values in the randomized trials of erythropoietin $[37,38]$. The Bayley evaluations are done in our clinical practice and parents can choose whether or not to complete them (65\% complete them). This means we may not capture the infants who are doing either extremely well or extremely poor, or parents who do not want to drive to the hospital or 
pay the co-pay. We were further limited by the COVID-19 pandemic. Overall, the lack of difference between cohorts is reassuring for the early use of budesonide, a potent corticosteroid, for the prevention of BPD.

The introduction of a change in a local standard of care requires careful planning and surveillance. A decision in the absence of an overwhelming quantity of data, revolves around when there is sufficient evidence to incorporate a new practice change into a local standard of care. The use of budesonide and surfactant had been evaluated in two randomized trials by Yeh et al. and demonstrated a larger change in BPD rates than any of the previous trials of noninvasive mechanical ventilation. In the setting of an institution with a higher rate of BPD, then local practice change based on available evidence can ethically be started. Similar to the introduction of early CPAP at many institutions prior to the results of SUPPORT and COIN trials [25], the local practice was changed to no longer give early surfactant without a CPAP trial even though it had yet been conclusively proven to be beneficial. Like with all clinical practice changes, ongoing surveillance for efficacy and safety are important to ensure that the changes are positive without unintended effects. Our IRB was consulted when we realized our practice change may have interest to an audience outside of our institution. They approved this study after determining that it could be done in a retrospective manner using de-identified data and a waiver of consent. Each institution considering changes in practice should utilize similar methods when conducting a retrospective study of a clinical practice change. One should also consider a possible publication bias, where NICUs with negative results choose not publish them. Larger randomized trials with budesonide, listed above, are underway and will give more definitive answers about this promising new treatment.

There are multiple limitations to the follow-up of our observational study. Since we are a large referral center, some of the infants do not return to follow-up clinic or return to follow-up in their local areas. We were limited to only $64 \%$ of patients following up for Bayley exams. Similar follow-up was seen between groups and demographics were similar between groups. Unfortunately, the COVID-19 pandemic forced us to close all in person Bayley testing, leading to only 54\% follow-up in the surfactant and budesonide cohort prior to the infants becoming out of the CA range. Six of the missed infants had telemedicine evaluations using the Vineland Adaptive Behavior Scales-Third Edition, and these results were similar to the cohort as whole. Our Bayley testing on average was performed in the younger portion of the age range (18.9 months CA) for the testing and thus may not compare with the 18-22 month CA range in many studies.
Since there were no differences in the ages between the cohorts, the lack of a difference at 18 months CA would likely remain at later time points as well. Only a small fraction of infants at 8-10 months CA received a Peabody exam, as our clinic protocols do not typically have the Physical Therapists see them at this time point, so the statistical significance of correlations was lower in this age group. The Peabody exams are primarily completed to identify infants needing early intervention services, and were not completed in a research protocol. Other important long-term morbidities, such as cerebral palsy, deafness, or blindness, were not formally assessed in these infants. We were only able to capture the Emergency Room visits and hospitalizations within the SSM Health system, so infants could have gone to other facilities. The rate of albuterol prescription could be due to changes in prescriber habits over time. We have used similar guidance for respiratory management,intubation/extubation, and non-invasive ventilation since 2012, but small changes could have occurred [39]. As these were observational cohorts over multiple years, other confounders could exist. Even with these limitations, we did not find trends for differences between the two cohorts.

\section{Conclusions}

In the developmental follow-up of our observational study of infants exposed to intratracheal budesonide for the reduction of BPD [10], the infants who received budesonide had similar fine and gross motor skills at 4-6 months CA, similar muscle tone on physician exams at 6 months CA, similar scores at 18-22 months CA on the Bayley III, and less prescriptions for albuterol in the first 2 years, when compared to infants who received surfactant alone. There were only minimal correlations between Peabody II motor evaluations at 4-6 months CA with Bayley III developmental scores at 18 months CA. In this cohort of infants with less severe RDS than the Yeh trials [9], the addition of budesonide to surfactant at birth was associated with a lower severity of BPD and without differences in neurologic outcomes at 6 months or 2 years CA.

Acknowledgements Grant Support: Department of Pediatrics and SSM Cardinal Glennon Foundation.

Author contributions Drs. Anderson and Hillman conceptualized and designed the study, extracted the data, analyzed the data, drafted the initial manuscript, and reviewed and revised the manuscript. Drs. Sadiq, Kothe, Josephsen, and Burleyson conceptualized and designed the study, extracted the data, and reviewed and revised the manuscript. Mr. Williams designed the study, extracted the data, and reviewed and revised the manuscript. All authors approved the final manuscript as submitted 


\section{Compliance with ethical standards}

Conflict of interest The authors declare no competing interests.

Publisher's note Springer Nature remains neutral with regard to jurisdictional claims in published maps and institutional affiliations.

\section{References}

1. Stoll BJ, Hansen NI, Bell EF, Walsh MC, Carlo WA, Shankaran S, et al. Trends in care practices, morbidity, and mortality of extremely preterm neonates, 1993-2012. JAMA. 2015;314:1039-51.

2. Horbar JD, Edwards EM, Greenberg LT, Morrow KA, Soll RF, Buus-Frank ME, et al. Variation in performance of neonatal intensive care units in the United States. JAMA Pediatr. 2017;171: e164396.

3. Johnson S, Moore T, Marlow N. Using the Bayley-III to assess neurodevelopmental delay: which cut-off should be used? Pediatr Res. 2014;75:670-4.

4. Laughon MM, Langer JC, Bose CL, Smith PB, Ambalavanan N, Kennedy KA, et al. Prediction of bronchopulmonary dysplasia by postnatal age in extremely premature infants. Am J Respir Crit Care Med. 2011;183:1715-22.

5. Ambalavanan N, Carlo WA, D'Angio CT, McDonald SA, Das A, Schendel D, et al. Cytokines associated with bronchopulmonary dysplasia or death in extremely low birth weight infants. Pediatrics. 2009;123:1132-41.

6. Baud O, Maury L, Lebail F, Ramful D, El Moussawi F, Nicaise C, et al. Effect of early low-dose hydrocortisone on survival without bronchopulmonary dysplasia in extremely preterm infants (PREMILOC): a double-blind, placebo-controlled, multicentre, randomised trial. Lancet. 2016;387:1827-36.

7. Doyle LW, Cheong JL, Ehrenkranz RA, Halliday HL. Early (< 8 days) systemic postnatal corticosteroids for prevention of bronchopulmonary dysplasia in preterm infants. Cochrane Database Syst Rev. 2017:10:CD001146.

8. Bassler D, Shinwell ES, Hallman M, Jarreau PH, Plavka R, Carnielli V, et al. Long-term effects of inhaled budesonide for bronchopulmonary dysplasia. N. Engl J Med. 2018;378:148-57.

9. Yeh TF, Chen CM, Wu SY, Husan Z, Li TC, Hsieh WS, et al. Intratracheal administration of budesonide/surfactant to prevent bronchopulmonary dysplasia. Am J Respir Crit Care Med. 2016;193:86-95.

10. Kothe TB, Sadiq FH, Burleyson N, Williams HL, Anderson C, Hillman NH. Surfactant and budesonide for respiratory distress syndrome: an observational study. Pediatr Res. 2020;87:940-5.

11. Bassler D, Plavka R, Shinwell ES, Hallman M, Jarreau PH, Carnielli V, et al. Early inhaled budesonide for the prevention of bronchopulmonary dysplasia. N. Engl J Med. 2015;373:1497-506.

12. Shinwell ES, Portnov I, Meerpohl JJ, Karen T, Bassler D. Inhaled corticosteroids for bronchopulmonary dysplasia: a meta-analysis. Pediatrics. 2016;138:1-10.

13. van den Brink KI, Boorsma M, Staal-van den Brekel AJ, Edsbacker S, Wouters EF, Thorsson L. Evidence of the in vivo esterification of budesonide in human airways. Br J Clin Pharmacol. 2008;66:27-35.

14. Brattsand R, Miller-Larsson A. The role of intracellular esterification in budesonide once-daily dosing and airway selectivity. Clin therapeutics. 2003;25:C28-41.

15. Huang LT, Yeh TF, Kuo YL, Chen PC, Chen CM. Effect of surfactant and budesonide on the pulmonary distribution of fluorescent dye in mice. Pediatrics Neonatol. 2015;56:19-24.
16. Ricci F, Catozzi C, Ravanetti F, Murgia X, D’Alo F, Macchidani $\mathrm{N}$, et al. In vitro and in vivo characterization of poractant alfa supplemented with budesonide for safe and effective intratracheal administration. Pediatr Res. 2017;82:1056-63.

17. Li L, Yang C, Feng X, Du Y, Zhang Z, Zhang Y. Effects of intratracheal budesonide during early postnatal life on lung maturity of premature fetal rabbits. Pediatr Pulmonol. 2018;53: $28-35$.

18. Kothe TB, Royse E, Kemp MW, Schmidt A, Salomone F, Saito M, et al. Effects of budesonide and surfactant in preterm fetal sheep. Am J Physiol Lung Cell Mol Physiol. 2018;315:L193-L201.

19. Kothe TB, Kemp MW, Schmidt A, Royse E, Salomone F, Clarke MW, et al. Surfactant plus budesonide decreases lung and systemic inflammation in mechanically ventilated preterm sheep. Am J Physiol Lung Cell Mol Physiol. 2019;316:L888-L893.

20. Roberts JK, Stockmann C, Dahl MJ, Albertine KH, Egan E, Lin Z, et al. Pharmacokinetics of budesonide administered with surfactant in premature lambs: implications for neonatal clinical trials. Curr Clin Pharm. 2016;11:53-61.

21. Doyle LW, Halliday HL, Ehrenkranz RA, Davis PG, Sinclair JC. An update on the impact of postnatal systemic corticosteroids on mortality and cerebral palsy in preterm infants: effect modification by risk of bronchopulmonary dysplasia. J Pediatr. 2014;165:1258-60.

22. Brouwer MJ, Kersbergen KJ, van Kooij BJM, Benders M, van Haastert IC, Koopman-Esseboom C, et al. Preterm brain injury on term-equivalent age MRI in relation to perinatal factors and neurodevelopmental outcome at two years. PLoS One. 2017;12: e0177128.

23. Rogers EE, Hintz SR. Early neurodevelopmental outcomes of extremely preterm infants. Semin Perinatol. 2016;40:497-509.

24. Claassen CC, Hillman NH, Brown K, Williams HL, Strand ML. Comparison of bubble CPAP devices using RAM cannula for extubation failure in very low birth weight infants: randomized and cohort studies. Neonatology. 2019;115:28-35.

25. Finer NN, Carlo WA, Walsh MC, Rich W, Gantz MG, Laptook AR, et al. Early CPAP versus surfactant in extremely preterm infants. N. Engl J Med. 2010;362:1970-9.

26. Ehrenkranz RA, Walsh MC, Vohr BR, Jobe AH, Wright LL, Fanaroff AA, et al. Validation of the National Institutes of Health consensus definition of bronchopulmonary dysplasia. Pediatrics. 2005;116:1353-60.

27. Abman SH, Collaco JM, Shepherd EG, Keszler M, Cuevas-Guaman $\mathrm{M}$, Welty SE, et al. Interdisciplinary care of children with severe bronchopulmonary dysplasia. J Pediatr. 2017;181:12-28. e11

28. Higgins RD, Jobe AH, Koso-Thomas M, Bancalari E, Viscardi RM, Hartert TV, et al. Bronchopulmonary dysplasia: executive summary of a workshop. J Pediatr. 2018;197:300-8.

29. Quinn GE, Ying GS, Bell EF, Donohue PK, Morrison D, Tomlinson LA, et al. Incidence and early course of retinopathy of prematurity: secondary analysis of the postnatal growth and retinopathy of prematurity (G-ROP) study. JAMA Ophthalmol. 2018;136:1383-9.

30. Connolly BH, McClune NO, Gatlin R. Concurrent validity of the Bayley-III and the Peabody Developmental Motor Scale-2. Pediatr Phys Ther. 2012;24:345-52.

31. Schmidt B, Roberts RS, Davis PG, Doyle LW, Asztalos EV, Opie $\mathrm{G}$, et al. Prediction of late death or disability at age 5 years using a count of 3 neonatal morbidities in very low birth weight infants. J Pediatr. 2015;167:982-6. e982

32. Connolly BH, Dalton L, Smith JB, Lamberth NG, McCay B, Murphy W. Concurrent validity of the Bayley Scales of Infant Development II (BSID-II) Motor Scale and the Peabody Developmental Motor Scale II (PDMS-2) in 12-month-old infants. Pediatr Phys Ther. 2006;18:190-6. 
33. Gill K, Osiovich A, Synnes A, Agnew JA, Grunau RE, Miller SP, et al. Concurrent validity of the Bayley-III and the Peabody Developmental Motor Scales-2 at 18 months. Phys Occup Ther Pediatr. 2019;39:514-24.

34. Spittle A, Orton J, Anderson PJ, Boyd R, Doyle LW. Early developmental intervention programmes provided post hospital discharge to prevent motor and cognitive impairment in preterm infants. Cochrane Database Syst Rev. 2015: CD005495.

35. Veldman SLC, Santos R, Jones RA, Sousa-Sa E, Okely AD. Associations between gross motor skills and cognitive development in toddlers. Early Hum Dev. 2019;132:39-44.

36. Anderson PJ, De Luca CR, Hutchinson E, Roberts G, Doyle LW, Victorian Infant Collaborative G. Underestimation of developmental delay by the new Bayley-III Scale. Arch Pediatr Adolesc Med. 2010;164:352-6.

37. Adams-Chapman I, Heyne RJ, DeMauro SB, Duncan AF, Hintz SR, Pappas A, et al. Neurodevelopmental impairment among extremely preterm infants in the neonatal research network. Pediatrics. 2018; 141:1-11.

38. Juul SE, Comstock BA, Wadhawan R, Mayock DE, Courtney SE, Robinson $\mathrm{T}$, et al. A randomized trial of erythropoietin for neuroprotection in preterm infants. N. Engl J Med. 2020;382:233-43.

39. Claassen CC, Strand ML, Williams HL, Hillman NH. Use of the RAM cannula with early bubble continuous positive airway pressure requires higher pressures: clinical and in vitro evaluations. Am J Perinatol. 2020. Online ahead of print. 